\title{
Gluon dynamics, center symmetry, and the deconfinement phase transition in SU(3) pure Yang-Mills theory
}

\author{
P. J. Silva* and O. Oliveira ${ }^{\dagger}$ \\ CFisUC, Department of Physics, University of Coimbra, P-3004 516 Coimbra, Portugal
}

(Received 8 January 2016; published 16 June 2016)

\begin{abstract}
The correlations between the modulus of the Polyakov loop, its phase $\theta$, and the Landau gauge gluon propagator at finite temperature are investigated in connection with the center symmetry for pure YangMills SU(3) theory. In the deconfined phase, where the center symmetry is spontaneously broken, the phase of the Polyakov loop per configuration is close to $\theta=0, \pm 2 \pi / 3$. We find that the gluon propagator form factors associated with $\theta \approx 0$ differ quantitatively and qualitatively from those associated to $\theta \approx \pm 2 \pi / 3$. This difference between the form factors is a property of the deconfined phase and a sign of the spontaneous breaking of the center symmetry. Furthermore, given that this difference vanishes in the confined phase, it can be used as an order parameter associated to the deconfinement transition. For simulations near the critical temperature $T_{c}$, the difference between the propagators associated to $\theta \approx 0$ and $\theta \approx \pm 2 \pi / 3$ allows one to classify the configurations as belonging to the confined or deconfined phase. This establishes a selection procedure which has a measurable impact on the gluon form factors. Our results also show that the absence of the selection procedure can be erroneously interpreted as lattice artifacts.
\end{abstract}

DOI: 10.1103/PhysRevD.93.114509

\section{INTRODUCTION}

The investigation of how the dynamics of QCD is modified by temperature and density has been under intensive study, motivated mainly by the experimental heavy-ion programs running at CERN [1] and RHIC [2]. From the theoretical side, the understanding of the phase diagram of QCD requires the extension of the usual theoretical toolkit to address the properties of strong interacting matter.

The simulations of QCD on a spacetime lattice provides $a b$ initio first principles results on the nonperturbative regime of hadronic phenomena. Lattice QCD simulations are routinely used to investigate the zero-temperature and zero-density properties of hadronic matter, to tackle the temperature dependence of the thermodynamic properties of hadrons, and to access the thermodynamics of hadronic matter at small densities; see, for example, Refs. [3,4] and references therein.

For pure SU(3) Yang-Mills theory and at zero density, lattice QCD simulations have shown the existence of a firstorder transition with the gluons becoming deconfined above the critical temperature $T_{c} \approx 270 \mathrm{MeV}$ [5-7]. For temperatures above $T_{c}$ the gauge bosons behave as massive quasiparticles and it is possible to define a gluon mass. Lattice simulations show that the gluon mass has a value of about $0.5 \mathrm{GeV}$ for temperatures around $T_{c}$ and its value increases linearly with $T$ [8]. On the other hand, for $T<T_{c}$ gluons are confined within color-singlet states. If for pure

\footnotetext{
* Corresponding author. psilva@uc.pt

†rlando@fis.uc.pt
}

gauge theory the singlet color states are glueballs, in the full theory other gauge-invariant states are possible as, e.g., mesons or baryons. If one takes into account the quark degrees of freedom, the picture just described is essentially unchanged. However, in such a case we have a crossover $[9,10]$ instead of a first-order transition to the deconfined phase, and the critical temperature is lowered to $T_{c} \approx$ $150 \mathrm{MeV}[11,12]$.

In what concerns the deconfinement phase transition, its order parameter is the Polyakov loop defined, in the continuum and in the Euclidean space, as

$$
L(\vec{x})=\frac{1}{N} \operatorname{Tr}\left\{\mathcal{P} \exp \left[i g \int_{0}^{1 / T} d x_{4} A_{4}(x)\right]\right\},
$$

where $\mathcal{P}$ stands for path ordering, $T$ is the temperature, and $N=3$ is the number of colors. Its space-averaged value

$$
L=\langle L(\vec{x})\rangle \propto e^{-F_{q} / T}
$$

is a measure of the free energy of a static quark $F_{q}$ [13]. In the confined phase, i.e., for $T<T_{c}, L=0$ and the quark free energy is infinite, suggesting that quarks are confined within hadrons. On the other hand, above $T_{c}$ the Polyakov loop is equal to one, which means that $F_{q}$ vanishes and quarks behave essentially as free particles; see, for example, Ref. [14] and references therein.

The Polyakov loop depends directly only on the glue content of the theory, but it distinguishes if quarks are confined or behave as quasi-free particles. In what concerns the glue content of QCD, there is not such an analogous operator. To the best of our knowledge, there is not an 
operator from which one can read about the nature of the gluons, i.e., if they are confined or behave as quasi-free particles. As observed in Refs. [8,15], the properties of the propagator change dramatically when $T$ crosses the critical temperature, and at least the so-called gluon electric form factor can be mapped into a free-particle propagator over a limited range of momenta for temperatures above $T_{c}$. On the other hand, the gluon magnetic form factor is clearly not compatible with the usual free-particle propagator.

The lattice definition of the Polyakov loop reads

$$
L(\vec{x})=\prod_{t=0}^{N_{t}} \mathcal{U}_{4}(\vec{x}, t),
$$

where $\mathcal{U}_{4}$ is the time-oriented link, and $L$ has the same definition as in the continuum formulation.

In QCD, like in any gauge theory, the gauge fields belong to the algebra of the gauge group and the fields related by a gauge transformation

$$
A_{\mu}^{\prime}(x)=G(x) A_{\mu}(x) G^{\dagger}(x)-\frac{i}{g} \partial_{\mu} G(x) G^{\dagger}(x)
$$

[where $G(x) \in S U(3)$ and $g$ is the coupling constant] are physically indistinguishable. The set of gauge related fields is called a gauge orbit. Choosing a gauge requires picking a given configuration from each gauge orbit. The choice of the gauge configurations on each gauge orbit is a delicate issue that has not been completely resolved in gauge theories; see, e.g., Refs. [16,17] and references therein. It is known that this choice of the gauge configuration can change the infrared properties of the theory [18-22].

For the group $\mathrm{SU}(3)$ one defines its center group

$$
Z_{3}=\left\{1, e^{i 2 \pi / 3}, e^{-i 2 \pi / 3}\right\},
$$

whose elements are such that they commute with all elements of the SU(3) group. The elements of $Z_{3}$, associated with global gauge transformations, divide the group $\mathrm{SU}(3)$ into equivalent classes. The gauge group associated with the pure Yang-Mills theory is $S U(3) / Z_{3}$ and not $\mathrm{SU}(3)$. In full QCD, the theory is not invariant under the replacement of $q(x) \rightarrow z q(x)$, where $z \in Z_{3}$ and, therefore, the gauge group associated with full QCD is $\mathrm{SU}(3)$.

The difference in the gauge group associated to the pure SU(3) Yang-Mills theory and full QCD implies, for example, that monopole solutions of the classical equations of motion exist only in the pure gauge theory; see, for example, Ref. [23] for further details.

For pure Yang-Mills theory the global gauge transformations associated with $Z_{3}$ leave unchanged the Green's function generating functional. This invariance occurs both for the continuum and the lattice formulation of QCD. From the elements of $Z_{3}$, it is possible to build gauge transformations which leave the generating functional invariant, but not the Polyakov loops.

Let us consider the lattice formulation of the pure $\mathrm{SU}(3)$ gauge theory. The Wilson action and the measure are invariant under a center transformation where the links on a given hyperplane $x_{4}=$ const are multiplied by some $z \in Z_{3}$. This type of transformation can be viewed as a singular gauge transformation. On the other hand, the Polyakov loop changes according to $L(\vec{x}) \rightarrow z L(\vec{x})$. In the confined phase where $L=0$, the space-averaged Polyakov loop is invariant under such center transformations. However, above the critical temperature, $L \neq 0$ and $L$ acquires a phase under the center transformation. Above the critical temperature $L$ is no longer invariant under a center transformation and the center symmetry is said to be spontaneously broken. Indeed, the simulations performed in Refs. [24-27] show that (i) the phase of $L$ takes values which match essentially those of the $Z_{3}$ elements, (ii) below $T_{c}$ the various phases of $L$ are equally populated, (iii) above $T_{c}$ the various phases of $L$ are not similarly populated, (iv) above $T_{c}$ one can identify center domains on the lattice, where the phases of $L(\vec{x})$ are close to a given $Z_{3}$ element, and (v) above $T_{c}$ these center domains define large clusters of $L$ that percolate the lattice volume. In Ref. [28] it was argued that, above $T_{c}$, the formation of the center domains can explain certain features of the quark-gluon plasma observed experimentally.

From the above considerations it follows that, on the lattice, for temperatures higher than $T_{c}$ one can label a given configuration by the phase of $L$. Furthermore, given a particular gauge configuration, the center symmetry allows one to generate a second configuration which-from the point of view of the sampling-is equally as probable as the original configuration. Indeed, although $L$ acquires a different phase, the action is exactly the same for both configurations.

If for $T>T_{c}$ the gauge configurations can be labeled by the phases of $L$, how different are the physical properties that can be associated with such equally probable configurations? Can we distinguish the various center domains? If, for example, the thermodynamics associated with the various $Z_{3}$ related configurations differ, do they lead to the formation of metastable states? The possibility of having new metastable states (depending on the mean lifetime of these states) has the potential to change our view of, for example, the history of the Universe.

The relevance of the pure Yang-Mills Euclidean metastable states mentioned in the previous paragraph to the physics as defined in Minkowski space is not clear. Indeed, according to some authors $[29,30]$ these states are relevant only to the computation of the Green's function generating functional in Euclidean space but do not lead to physical domains in Minkowski space. In what concerns the role of the above-mentioned metastable states, a definitive answer requires a proper understanding of the analytical 
continuation of quantum field theory from Minkowski to Euclidean space and vice versa beyond perturbation theory.

Early studies of the correlation between chiral symmetry breaking and the phase of the Polyakov loop can be found in Refs. [31-33]. According to Ref. [33], in the deconfined phase, the breaking of chiral symmetry is correlated with the phase of $L$. If chiral symmetry breaking is sensitive to the phase of the Polyakov loop, are there other properties of QCD which are also correlated with the phase of the Polyakov loop?

In the present work we try to identify similar effects in pure gauge theory. In particular, we try to correlate the gluon propagator, computed using lattice QCD simulations, with the phase of the Polyakov loop. Our results show that, for temperatures above $T_{c}$, the gluon propagator is quantitatively and qualitatively different for the various $Z_{3}$ related configurations. Furthermore, we find that the propagator associated with the $e^{ \pm i 2 \pi / 3}$ phases for the Polyakov loop are indistinguishable, within our statistical precision. We also observe that the values of the phase of the Polyakov loop are correlated with the infrared behavior of the longitudinal gluon propagator, in particular with its value at zero momentum. Moreover, these correlations can be used to identify the deconfinement phase transition relying only on the gluon propagator. Preliminary results of our work can be found in Ref. [34].

In the literature there are several studies of the lattice Landau gauge gluon propagator at finite temperature $[8,15,35-47]$, both for $\mathrm{SU}(2)$ and $\mathrm{SU}(3)$ gauge theories. However, to the best of our knowledge, no one has investigated the correlations of the propagator with the phase of the Polyakov loop. Continuous methods have also been applied to the study of the temperature dependence of the Landau gauge gluon propagator (see Refs. [48-52] and references therein), but again the correlation with the phase of the Polyakov loop was not taken into account.

This paper is organized as follows. In Sec. II the lattice setup, and the computation of the gluon field and the gluon propagator are discussed. Furthermore, in this section the $Z_{3}$ sectors are introduced and the dependence of the gluon propagator with the phase of the Polyakov loop is reported at a temperature well above the critical temperature. In Sec. III we discuss the simulations close to the critical temperature and identify a criterion to determine the phase (confined or deconfined) of a given configuration in a Monte Carlo simulation. In Sec. IV the behavior of the gluon propagator near $T_{c}$ is investigated, together with a brief discussion of the continuum limit. Finally, in Sec. V we resume and conclude.

\section{LATTICE SETUP, GLUON PROPAGATOR, CENTER SYMMETRY, AND $Z_{3}$ SECTORS}

In the present work one considers various lattice simulations using the Wilson gauge action for the gauge group $\mathrm{SU}(3)$ and for different lattice spacings, i.e., $\beta$ values. The physical scale used to convert into physical units was taken from the string tension, following the procedure described in Ref. [8].

The simulations were performed on several asymmetric lattices $L_{s}^{3} \times L_{t}$ with a physical spatial volume $\sim(6.5 \mathrm{fm})^{3}$ and $L_{t}=6,8$. We take the inverse of the lattice time extension $T=1 / L_{t}$ in physical units as the definition for temperature.

In order to illustrate the behavior of the various propagators at temperatures above $T_{c}$, we first report the results obtained on $64^{3} \times 6$ for $\beta=6.0 \quad(T=324 \mathrm{MeV})$. Furthermore, we will also investigate the results of simulations using two sets of lattices close to the critical temperature (see Table I): (i) a set of coarser lattices with a lattice spacing $a \sim 0.12 \mathrm{fm}$ and $\beta \sim 5.9$, and (ii) a second set of finer lattices with lattice spacing $a \sim 0.09 \mathrm{fm}$ and $\beta \sim 6.0$. Although the simulations with the coarser and finer lattices do not cover exactly the same range of temperatures, they will allow us to estimate the effect due to the use of a finite lattice spacing. Given the relatively large physical volumes $(6.49-6.68 \mathrm{fm})^{3}$, we hope that the finitevolume effects are small. Indeed, the studies of the gluon propagator at zero temperature [53] suggest that, for sufficiently large volumes, finite-volume effects do not significantly change the propagator. In the present work, due to finite computing resources, no attempts are made to look at Gribov copies effects; see, e.g., Refs. [18,21,22] and references therein for results at zero temperature.

TABLE I. The lattice setup. The physical scale was defined from the string tension. The values of $\beta$ were adjusted such that $L_{s} a \simeq 6.5-6.6 \mathrm{fm}$.

\begin{tabular}{lcccc}
\hline \hline Temp. $(\mathrm{MeV})$ & $L_{s}^{3} \times L_{t}$ & $\beta$ & $a(\mathrm{fm})$ & $L_{s} a(\mathrm{fm})$ \\
\hline 265.9 & $54^{3} \times 6$ & 5.890 & 0.1237 & 6.68 \\
266.4 & $54^{3} \times 6$ & 5.891 & 0.1235 & 6.67 \\
266.9 & $54^{3} \times 6$ & 5.892 & 0.1232 & 6.65 \\
267.4 & $54^{3} \times 6$ & 5.893 & 0.1230 & 6.64 \\
268.0 & $54^{3} \times 6$ & 5.8941 & 0.1227 & 6.63 \\
268.5 & $54^{3} \times 6$ & 5.895 & 0.1225 & 6.62 \\
269.0 & $54^{3} \times 6$ & 5.896 & 0.1223 & 6.60 \\
269.5 & $54^{3} \times 6$ & 5.897 & 0.1220 & 6.59 \\
270.0 & $54^{3} \times 6$ & 5.898 & 0.1218 & 6.58 \\
271.0 & $54^{3} \times 6$ & 5.900 & 0.1213 & 6.55 \\
272.1 & $54^{3} \times 6$ & 5.902 & 0.1209 & 6.53 \\
273.1 & $54^{3} \times 6$ & 5.904 & 0.1204 & 6.50 \\
269.2 & $72^{3} \times 8$ & 6.056 & 0.09163 & 6.60 \\
270.1 & $72^{3} \times 8$ & 6.058 & 0.09132 & 6.58 \\
271.0 & $72^{3} \times 8$ & 6.060 & 0.09101 & 6.55 \\
271.5 & $72^{3} \times 8$ & 6.061 & 0.09086 & 6.54 \\
271.9 & $72^{3} \times 8$ & 6.062 & 0.09071 & 6.53 \\
272.4 & $72^{3} \times 8$ & 6.063 & 0.09055 & 6.52 \\
272.9 & $72^{3} \times 8$ & 6.064 & 0.09040 & 6.51 \\
273.3 & $72^{3} \times 8$ & 6.065 & 0.09025 & 6.50 \\
273.8 & $72^{3} \times 8$ & 6.066 & 0.09010 & 6.49 \\
\hline \hline
\end{tabular}


The gauge configurations were generated with the Chroma library [54]. For the gauge fixing we use the Fourier accelerated steepest descent method described in Ref. [55], which was implemented using the Chroma and PFFT [56] libraries. For each gauge configuration, the lattice equivalent of the average value of $\left(\partial_{\mu} A_{\mu}^{a}\right)^{2}$ per site and color index (see Ref. [8] for details) was stopped when it reached a value smaller than $10^{-15}$.

For each of the ensembles reported in Table I, the gluon propagator was computed using 100 gauge configurations. For the generation of the links a combined Monte Carlo sweep of four Cabibbo-Marinari heat bath and seven overrelaxation sweeps was used; the measurements were performed every 100 combined sweeps after discarding the first 500 combined sweeps in the Markov chain for thermalization.

\section{A. The gluon propagator}

The computation of the gluon propagator requires a definition of the $A_{\mu}$ from the links. In the current work we take the usual expression

$$
a g_{0} A_{\mu}\left(x+\frac{a}{2} \hat{e}_{\mu}\right)=\frac{1}{2 i}\left[\mathcal{U}_{\mu}(x)-\mathcal{U}_{\mu}^{\dagger}(x)\right]_{\text {traceless }}
$$

where $\hat{e}_{\mu}$ is the unit vector along the lattice direction $\mu$ and $g_{0}$ is the bare coupling constant. The above definition assumes that the link and the gluon field are related by

$$
\begin{aligned}
\mathcal{U}_{\mu}(x) & =\exp \left(\operatorname{iag}_{0} A_{\mu}\left(x+\frac{a}{2} \hat{e}_{\mu}\right)\right) \\
& \approx 1+\operatorname{iag}_{0} A_{\mu}\left(x+\frac{a}{2} \hat{e}_{\mu}\right) .
\end{aligned}
$$

The above relations are certainly valid when one considers fluctuations around the trivial configuration, as in, e.g., perturbation theory. Given that we will look at configurations whose Polyakov loop is of the type $|L| e^{i \theta}$ with $\theta \approx 0, \pm 2 \pi / 3$, one might ask whether the above definition is still valid when $\theta= \pm 2 \pi / 3$.

In Fig. 1 we show the lattice average values of $A_{\mu}^{a}(x)$ for $a=0, \ldots, N_{c}^{2}-1, \mu=0 \ldots N_{d}-1$ for a $64^{3} \times 6$ configuration. In all cases, $\left\langle A_{\mu}^{a}\right\rangle$ is compatible with zero within one or two standard deviations for all color and Lorentz indices. Furthermore, apart from a scaling factor, there is no clear difference between the configurations associated to the different phases of the Polyakov loop. We take this result as an indication that the gluon field given by Eq. (5) can be applied to all configurations-including all possible values of the Polyakov loop-considered here.

A generalized connection between the link and the gluon field given by

$$
\mathcal{U}_{\mu}(x) \approx u_{0}\left[1+\operatorname{iag}_{0}\left(A_{\mu}\left(x+\frac{a}{2} \hat{e}_{\mu}\right)+a_{\mu}\right)\right]
$$

(where $u_{0}$ is a real number and $a_{\mu}$ are constant fields) could be used. However, the replacement of Eq. (6) by Eq. (7) gives the same bare gluon field up to a multiplicative
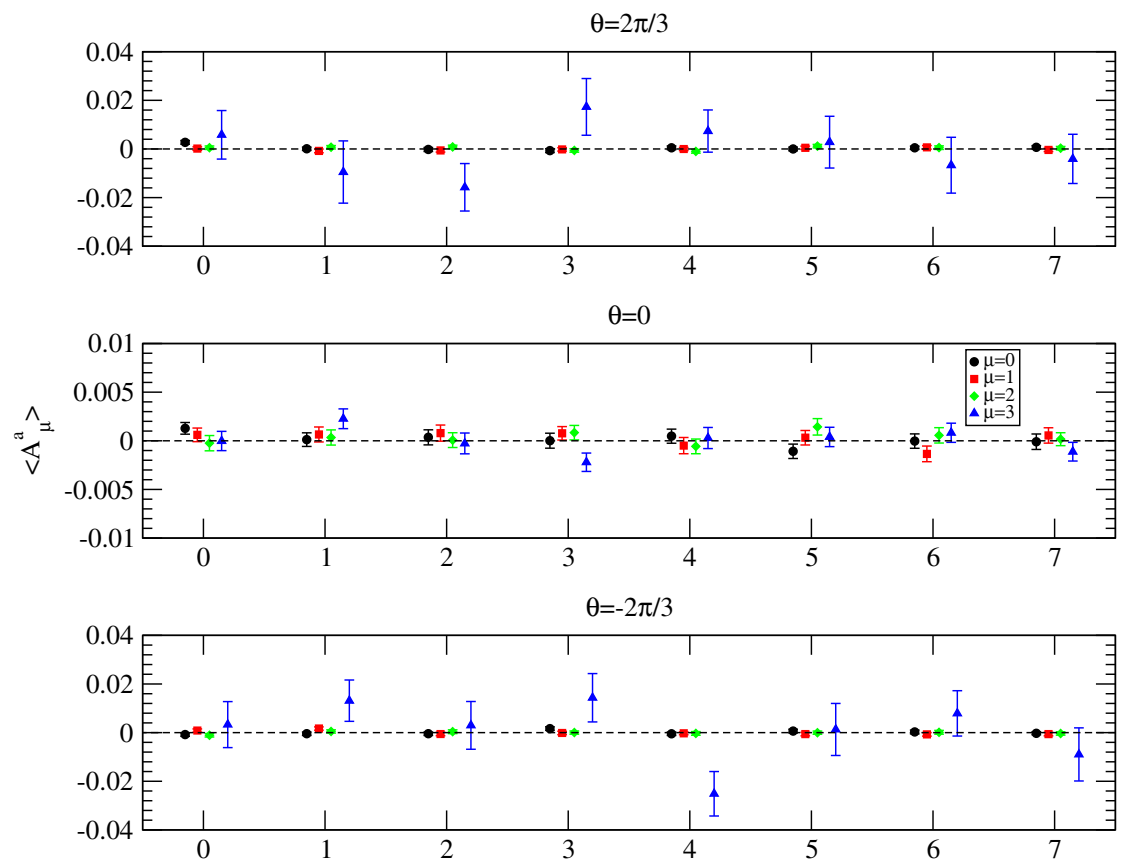

FIG. 1. Average values of $A_{\mu}^{a}(x)$ for a $64^{3} \times 6, \beta=6.0(T=324 \mathrm{MeV})$ configuration. 
constant and a different zero-momentum gluon field. In what concerns $u_{0}$, the use of a MOM scheme to renormalize the propagator removes any dependence on $u_{0}$. It follows that the differences of using Eq. (6) or Eq. (7) to compute the gluon propagator can only appear for the zeromomentum propagator, which leaves the main conclusions of the current work unchanged.

In the Landau gauge and at finite temperature, the gluon propagator reads

$$
\left\langle A_{\mu}^{a}(p) A_{\nu}^{b}(q)\right\rangle=V \delta^{a b} \delta(p+q) D_{\mu \nu}^{a b}(p),
$$

where

$$
D_{\mu \nu}^{a b}(p)=\delta^{a b}\left\{P_{\mu \nu}^{T} D_{T}\left(p_{4}, \vec{p}\right)+P_{\mu \nu}^{L} D_{L}\left(p_{4}, \vec{p}\right)\right\},
$$

and the transverse and longitudinal projectors are given by

$$
\begin{gathered}
P_{\mu \nu}^{T}=\left(1-\delta_{\mu 4}\right)\left(1-\delta_{\nu 4}\right)\left(\delta_{\mu \nu}-\frac{p_{\mu} p_{\nu}}{\vec{p}^{2}}\right), \\
P_{\mu \nu}^{L}=\left(\delta_{\mu \nu}-\frac{p_{\mu} p_{\nu}}{p^{2}}\right)-P_{\mu \nu}^{T} .
\end{gathered}
$$

In the above expressions, latin letters stand for color indices and greek letters for spacetime indices.

The results shown here are for renormalized longitudinal and transverse propagators. For the renormalization we follow the procedure devised in Ref. [8], taking $\mu=4 \mathrm{GeV}$ for the renormalization scale and setting $D_{L, T}\left(\mu^{2}\right)=Z_{R} D_{L, T}^{\mathrm{Lat}}\left(\mu^{2}\right)=1 / \mu^{2}$. The longitudinal and transverse form factors were renormalized independently within each possible phase value of the associated Polyakov loop. It turns out that, in our simulations, the renormalization constants for the longitudinal and transverse form factors agree within one standard deviation for all possible values of the phase.

\section{B. Center symmetry}

For pure Yang-Mills theory formulated on the lattice, the Wilson action and the path-integral measure are invariant under the SU(3) group center, i.e., under transformations of the type

$$
\mathcal{U}_{4}(\vec{x}, t=0) \rightarrow \mathcal{U}_{4}^{\prime}(\vec{x}, t=0)=z \mathcal{U}_{4}(\vec{x}, t=0)
$$

for all $z \in Z_{3}$. For temperatures below $T_{c}$ the center symmetry is preserved and the various phases of $L$ are similarly sampled, which implies $\langle L\rangle \sim 0$. Above the critical temperature, the center symmetry is spontaneously broken and the average value of $L$ over the lattice no longer vanishes.

As described in Refs. [24,25,27], for $T>T_{c}$ it is possible to identify center domains where the phase of the Polyakov loop is $\approx 0, \pm 2 \pi / 3$, i.e., it coincides with the

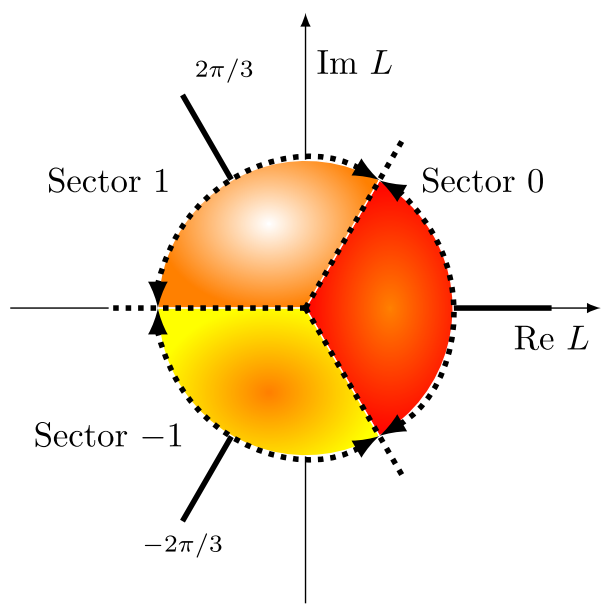

FIG. 2. The definition of the $Z_{3}$ sectors.

phase of the elements of $Z_{3}$. The dimensions of the center domains are temperature dependent and, at the critical temperature, these clusters percolate the lattice. In this sense, the gauge configurations can be classified according to the associated phase of the Polyakov loop,

$$
L=\langle L\rangle=|L| e^{i \theta} .
$$

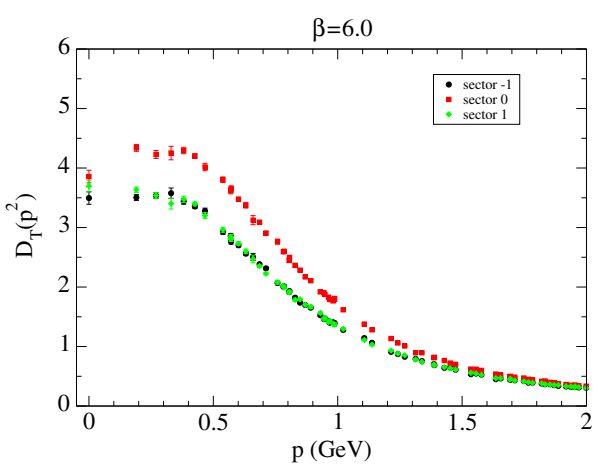

(a) Magnetic Form Factor

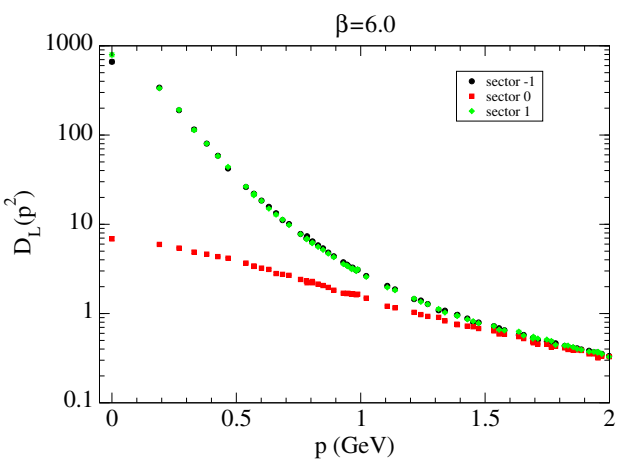

(b) Electric Form Factor

FIG. 3. Gluon propagators for the different sectors at $T=324 \mathrm{MeV}$. 


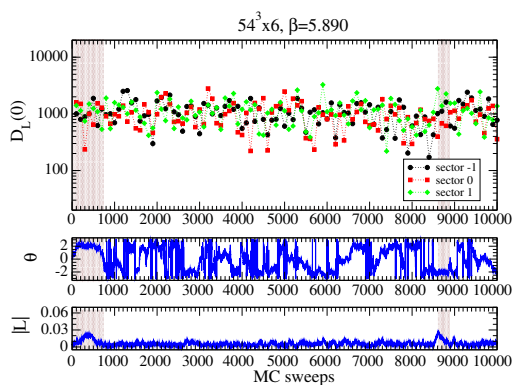

(a) $T=265.9 \mathrm{MeV}$

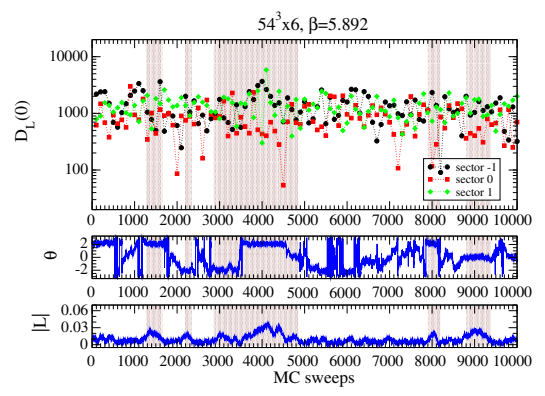

(c) $T=266.9 \mathrm{MeV}$

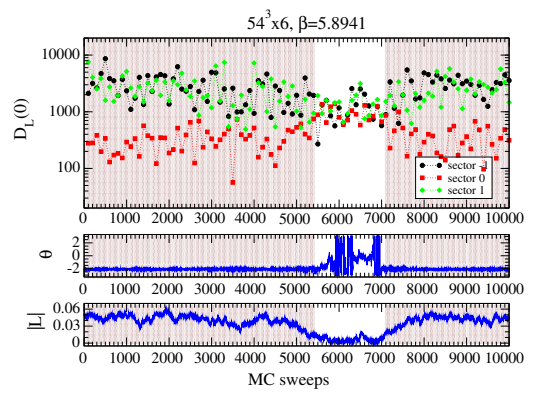

(e) $T=268 \mathrm{MeV}$

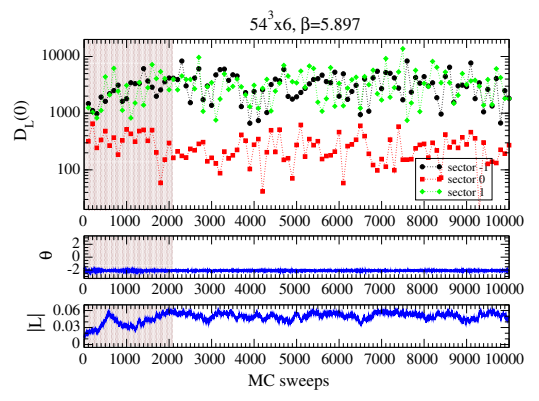

(g) $T=269.5 \mathrm{MeV}$

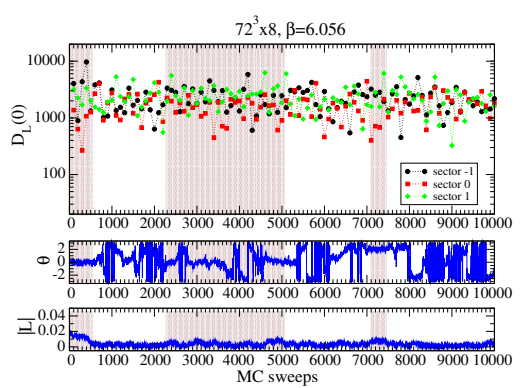

(b) $T=269.2 \mathrm{MeV}$

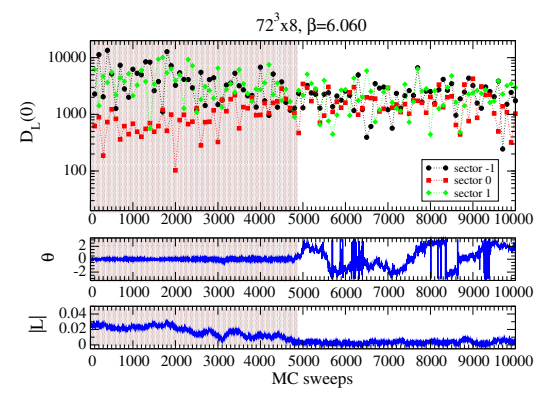

(d) $T=271 \mathrm{MeV}$

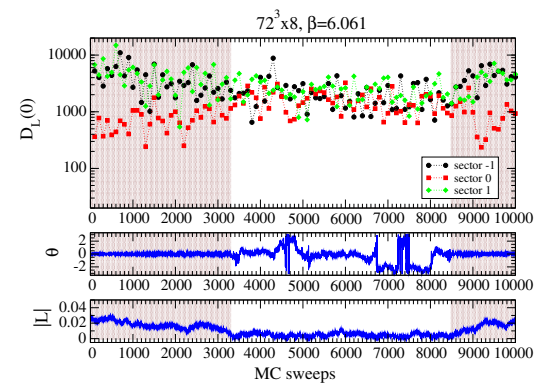

(f) $T=271.5 \mathrm{MeV}$

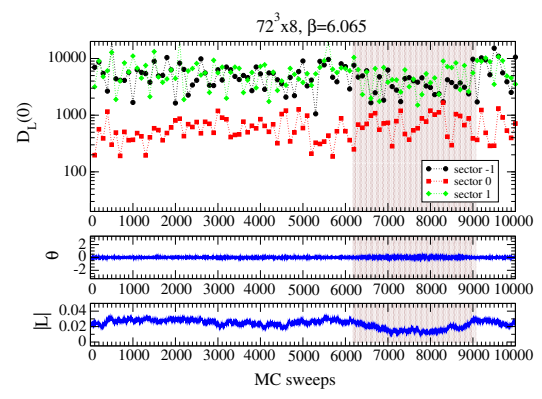

(h) $T=273.3 \mathrm{MeV}$

FIG. 4. Monte Carlo history of the coarse (left) and fine (right) ensembles for bare $|L|, \theta$, and $D_{L}(0)$. Configurations inside the colored areas will not be used to compute the propagators; see text for details.

Center transformations map configurations in different equivalent classes, i.e., with different $\theta$. We have performed a number of simulations using various volumes (results not shown here) and they suggest that, in the Markov chain, the probability for the transition between the equivalent classes decreases when the physical volume of the spatial lattice increases. This suggests that, in the limit of infinite volume, the sampling is confined to configurations whose Polyakov loop is such that $\theta$ takes one and only one value in $\{0, \pm 2 \pi / 3\}$.

\section{C. $Z_{3}$ sectors}

Our goal is to try to understand if the dynamics of the gluon field changes with the phase of the Polyakov loop. The transformations of the type (12) map configurations with the same action which, from the point of view of the sampling, belong to classes with exactly the same probability. In principle, one could include these transformations in the definition of the Markov process and, in this way, sample all the possible phases of the Polyakov loop equally. However, in this work the gauge configurations are 


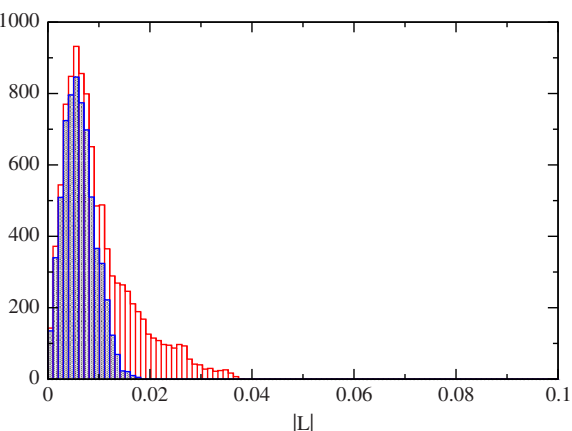

(a) coarser lattice for $T=266.9 \mathrm{MeV}$

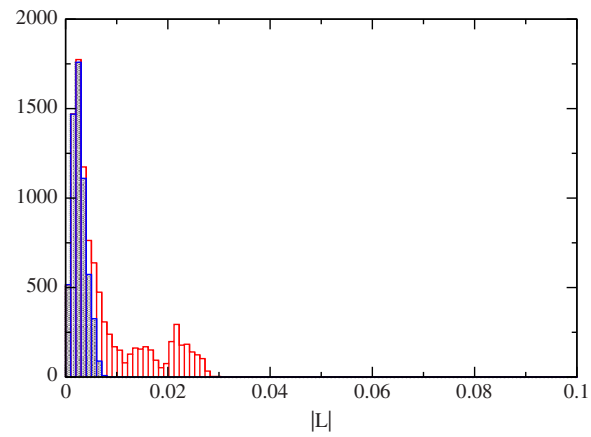

(c) Finer lattice for $T=270.1 \mathrm{MeV}$

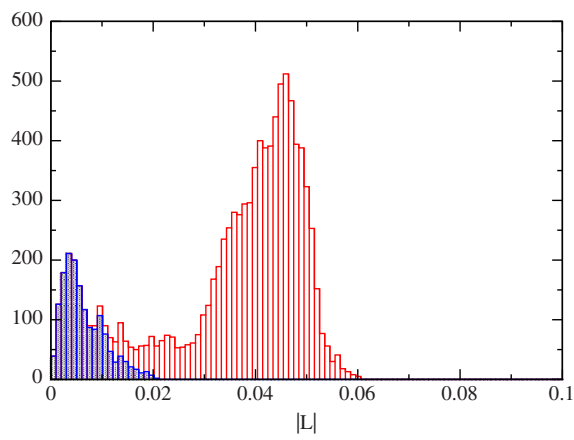

(b) coarser lattice for $T=268 \mathrm{MeV}$

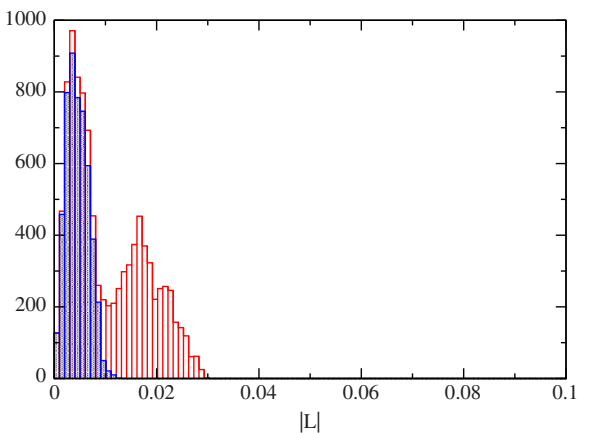

(d) Finer lattice for $T=271.5 \mathrm{MeV}$

FIG. 5. Histograms of $|L|$ for various $T$ before (red) and after the selection procedure (blue) for all independent configurations obtained during the sampling. See text for details.

generated in the usual way and, before gauge fixing, one applies transformations of the type (12) for all $z \in Z_{3}$ to each configuration. Then, each of these configurations is rotated to the (minimal) Landau gauge and classified according to the phase of the corresponding average value of the Polyakov loop $\langle L\rangle=|L| e^{i \theta}$ as

$$
\theta= \begin{cases}-\pi<\theta \leq-\frac{\pi}{3}, & \text { Sector }-1, \\ -\frac{\pi}{3}<\theta \leq \frac{\pi}{3}, & \text { Sector } 0, \\ \frac{\pi}{3}<\theta \leq \pi, & \text { Sector } 1 .\end{cases}
$$

This classification of the configurations is shown in Fig. 2. We recall that, for each gauge configuration, although the Polyakov loop's main contribution comes from center domains belonging to a given $Z_{3}$ sector, the other sectors are also present in smaller center domains.

In order to illustrate what happens to the gluon propagator in each of the $Z_{3}$ sectors, in Fig. 3 we show the electric and magnetic components of the propagator per $Z_{3}$ sector for $T=324 \mathrm{MeV}$ computed on a $64^{3} \times 6$ lattice for $\beta=6.0$. Figure 3 shows the typical behavior of the propagators for $T>T_{c}$. For temperatures close to $T_{c}$ a careful analysis is required (see the discussion below), but the pattern observed in Fig. 3 still applies if we approach the critical temperature from above.

If the electric form factor for the 0 sector is suppressed relative to the \pm 1 sectors, for the magnetic form factor the situation is reversed with the sector 0 being enhanced relative to the \pm 1 sectors. One can translate this result into a mass scale defined by the inverse of the square root of the propagator at zero momentum. The mass scale associated with the electric sector is much larger for the 0 sector, in comparison with the \pm 1 sectors. On the other hand, the mass associated with the magnetic sector is smaller for the 0 sector, in comparison with the \pm 1 sectors. From Fig. 3 one can identify the following mass hierarchy: $m_{L}( \pm 1)<m_{L}(0)<m_{M}(0)<m_{M}( \pm 1)$, where $m_{L}\left(m_{M}\right)$ stands for electric (magnetic) mass and the corresponding sectors are in parentheses.

\section{SIMULATIONS NEAR THE PHASE TRANSITION}

The differences observed in the gluon propagator form factors and reported in Fig. 3 seem to be a feature of the deconfined phase and happen for $T>T_{c}$. For the pure glue theory, the deconfinement phase transition is of first order. Near the critical temperature, Monte Carlo simulations access configurations in the confined and deconfined phases. The simulations we have performed for $T \approx T_{c} \approx$ $270 \mathrm{MeV}$ also show that the probability transition between the confined and deconfined phases in the Markov chain decreases when we increase the physical volume used to simulate the theory. In order to properly describe any of the two phases near $T_{c}$, one needs to define a way to separate the configurations belonging to either phase. 


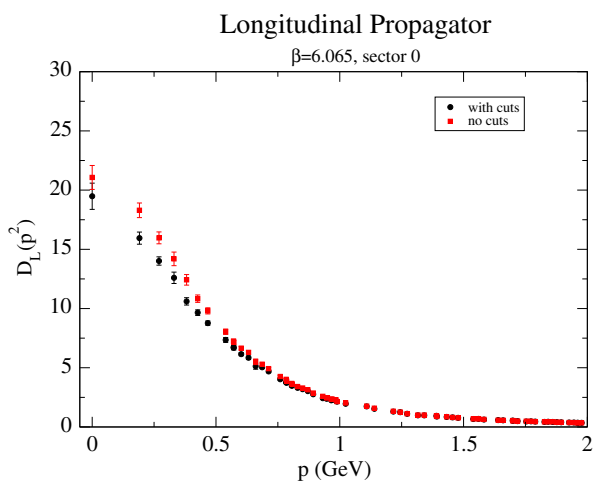

(a) $L_{s}=72, \beta=6.065$, sector 0 .

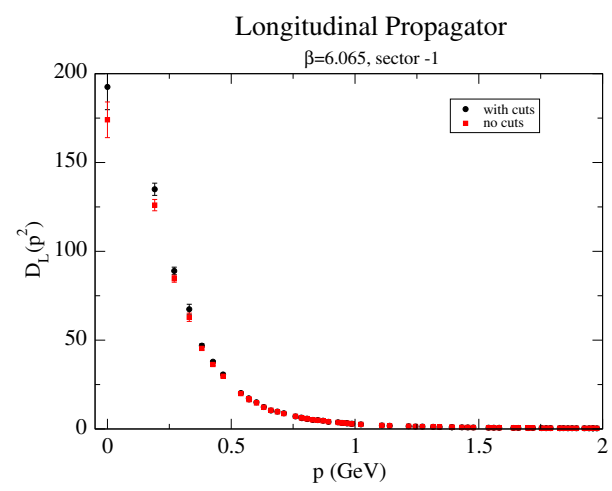

(b) $L_{s}=72, \beta=6.065$, sector -1 .

FIG. 6. Example of how the selection procedure changes the gluon electric form factor for the finer lattice and $T=273.3 \mathrm{MeV}$.

In Fig. 4 we report the Markov chain history for simulations performed with a temperature close to $T_{c}$ and for the coarser and finer lattices. The figure includes the corresponding values of the modulus of the bare Polyakov loop, its phase, and the longitudinal (electric) gluon form factor. For the simulations reported, the modulus of the bare Polyakov loop seems to take a continuous range of values. When it takes higher values, one observes that $D_{L}(0)$ computed for configurations in the \pm 1 sectors differs substantially from the propagator in the zero sector. On the other hand, when $|L|$ takes smaller

TABLE II. The same as Table I. The number of configurations refers to the configurations which, according to our selection procedure, will be used to compute the propagator. See text for details.

\begin{tabular}{lccc}
\hline \hline Temp. $(\mathrm{MeV})$ & $L_{s}^{3} \times L_{t}$ & $\beta$ & No. Configs \\
\hline 265.9 & $54^{3} \times 6$ & 5.890 & 90 \\
266.4 & $54^{3} \times 6$ & 5.891 & 91 \\
266.9 & $54^{3} \times 6$ & 5.892 & 65 \\
267.4 & $54^{3} \times 6$ & 5.893 & 76 \\
268.0 & $54^{3} \times 6$ & 5.8941 & 16 \\
268.5 & $54^{3} \times 6$ & 5.895 & 63 \\
269.0 & $54^{3} \times 6$ & 5.896 & 100 \\
269.5 & $54^{3} \times 6$ & 5.897 & 80 \\
270.0 & $54^{3} \times 6$ & 5.898 & 100 \\
271.0 & $54^{3} \times 6$ & 5.900 & 95 \\
272.1 & $54^{3} \times 6$ & 5.902 & 100 \\
273.1 & $54^{3} \times 6$ & 5.904 & 100 \\
269.2 & $72^{3} \times 8$ & 6.056 & 63 \\
270.1 & $72^{3} \times 8$ & 6.058 & 59 \\
271.0 & $72^{3} \times 8$ & 6.060 & 52 \\
271.5 & $72^{3} \times 8$ & 6.061 & 51 \\
271.9 & $72^{3} \times 8$ & 6.062 & 82 \\
272.4 & $72^{3} \times 8$ & 6.063 & 70 \\
272.9 & $72^{3} \times 8$ & 6.064 & 100 \\
273.3 & $72^{3} \times 8$ & 6.065 & 71 \\
273.8 & $72^{3} \times 8$ & 6.066 & 100 \\
\hline \hline
\end{tabular}

values, it follows that the propagators computed using configurations in any of the $Z_{3}$ sectors are indistinguishable. We associated the first type of configurations with the deconfined phase, while the latest family of configurations has been identified with the confined phase. Recall that in the results reported in Fig. 3, for temperatures well above the critical temperature, it was observed that $D_{L}(0)$ for the \pm 1 sectors is enhanced relative to the zero sector. Furthermore, the time evolution of $\theta$ also shows that for lower values of $|L|$, the phase of the Polyakov loop fluctuates freely, suggesting a $|L| \sim 0$ as expected in the confined phase.

The observed correlations in the time evolution of $|L|, \theta$, and the differences in $D_{L}(0)$ associated to the various $Z_{3}$ sectors suggests that this difference between the propagators can be used as a criterion to identify the phase (confined or deconfined) of a given configuration. In this spirit, the simulations performed on the coarser lattices with $\beta \leq 5.895(T \leq 268.5 \mathrm{MeV})$ or on finer lattices with $\beta \leq$ $6.061(T \leq 271.5 \mathrm{MeV})$ provide, in general, configurations in the confined phase. On the other hand, simulations with higher values of $\beta$ are mainly in the deconfined phase.

One can use the criterion discussed above to separate the configurations belonging to each phase (confined or deconfined) in a given simulation. In Fig. 4 we provide various examples of using our criteria. The color code used is such that the configurations belonging to the confined phase are plotted against a white background for simulations on coarser lattices with $\beta \leq 5.895$ and finer lattices with $\beta \leq 6.061$. The remaining configurations, i.e., those belonging to deconfined phase or not clearly in any of the phases, are plotted against a colored background. On the other hand, for those simulations with a higher $\beta$ and $T$ the white background identifies configurations in the deconfined phase, while the colored background refers to all the others. In this way, the configurations associated to the dominant phase in a given simulation are plotted against a white background. There is an exception to this rule: the color code used for the coarser lattice with $\beta=5.8941$ $(T=268 \mathrm{MeV})$. According to Ref. [7] the infinite-volume 


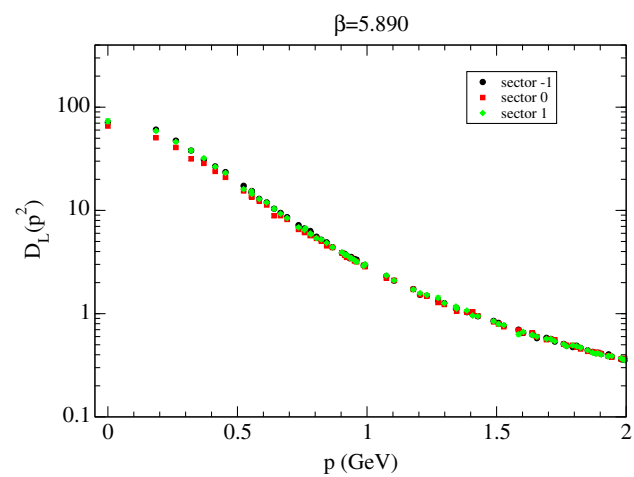

(a) $T=265.9 \mathrm{MeV}$

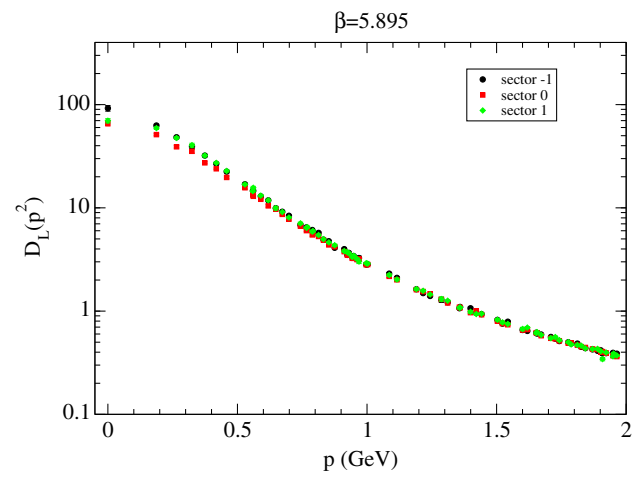

(c) $T=268.5 \mathrm{MeV}$

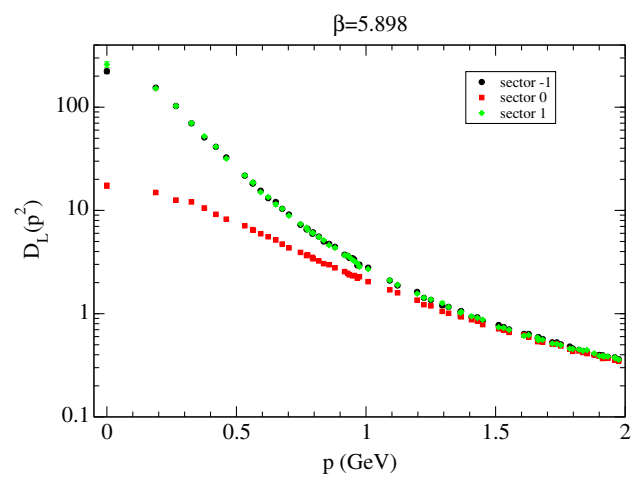

(e) $T=270.0 \mathrm{MeV}$

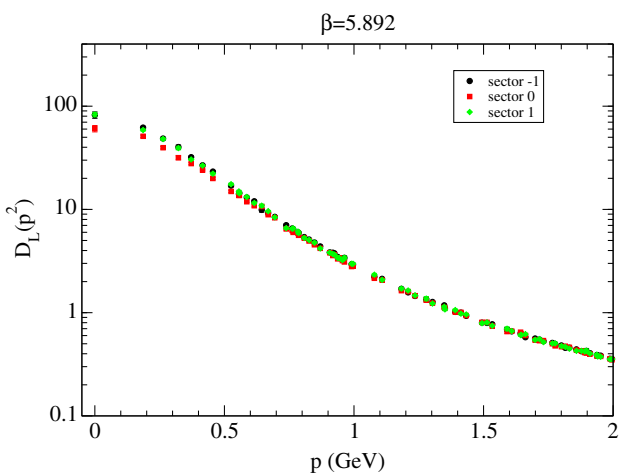

(b) $T=266.9 \mathrm{MeV}$

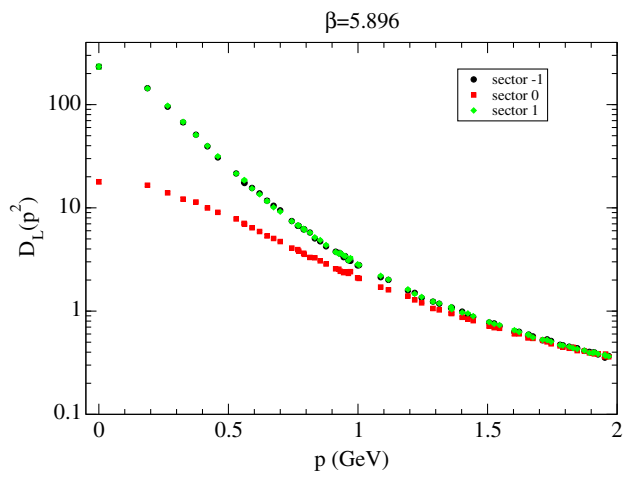

(d) $T=269.0 \mathrm{MeV}$

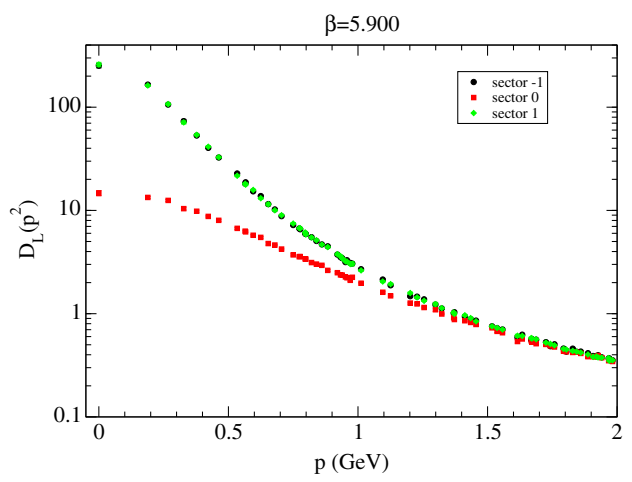

(f) $T=271.0 \mathrm{MeV}$

FIG. 7. Electric gluon form factor $D_{L}\left(p^{2}, T\right)$ for simulations using the coarser $54^{3} \times 6$ lattices.

extrapolation of the critical value for $\beta$ is 5.8941(12). Using the separation of $D_{L}(0)$ as a criterion to identify the dominant phase in a given simulation, it turns out that for $\beta=5.8941$ the majority of the configurations belong to the deconfined phase. Furthermore, for $\beta=$ $5.895(T=268.5 \mathrm{MeV})$ the configurations are either on the confined phase or do not clearly belong to any of the phases. Further, for $\beta=5.896(T=269 \mathrm{MeV})$ all the configurations belong to the deconfined phase. This is an indication that the infinite-volume limit was not achieved, although we expect the finite-volume effects to be small. If one wants to report a continuous and smooth behavior, we have to take for $\beta=5.8941$ only those configurations which belong to the confined phase. Therefore, in Fig. 4 the color code for $\beta=5.8941$ is reversed.

The use of the separation of $D_{L}(0)$ to identify confined and deconfined configurations in the Monte Carlo time history allows an estimate of $T_{c}$. The simulations point towards a $T_{c}$ in the range 269-272 MeV, in good accordance with the literature.

In order to illustrate the effects of the selection procedure just described, in Fig. 5 we show the histograms of the modulus of the Polyakov loop before (red lines) and after 


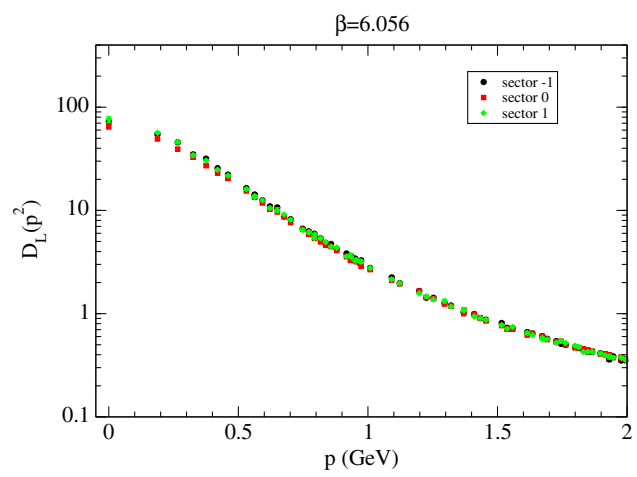

(a) $T=269.2 \mathrm{MeV}$

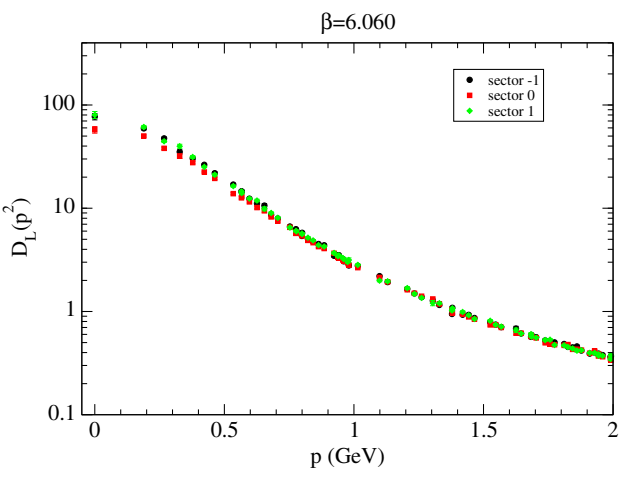

(c) $T=271.0 \mathrm{MeV}$

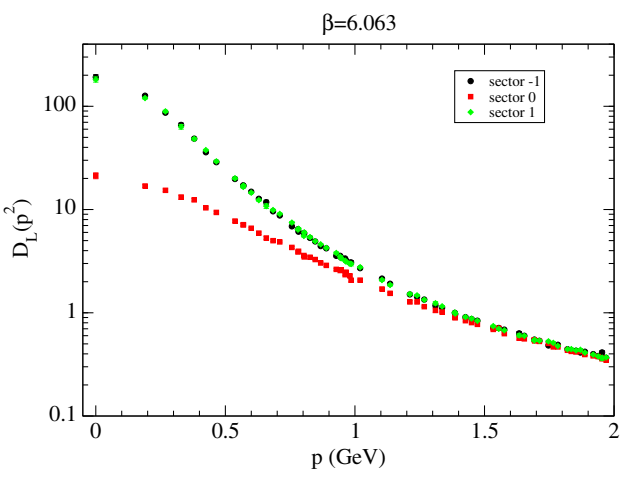

(e) $T=272.4 \mathrm{MeV}$

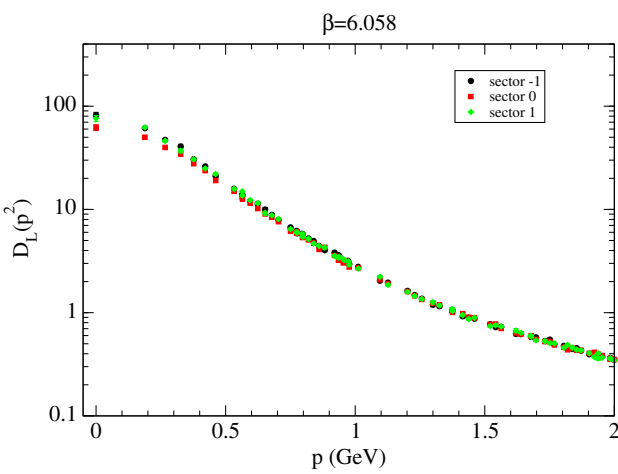

(b) $T=270.1 \mathrm{MeV}$

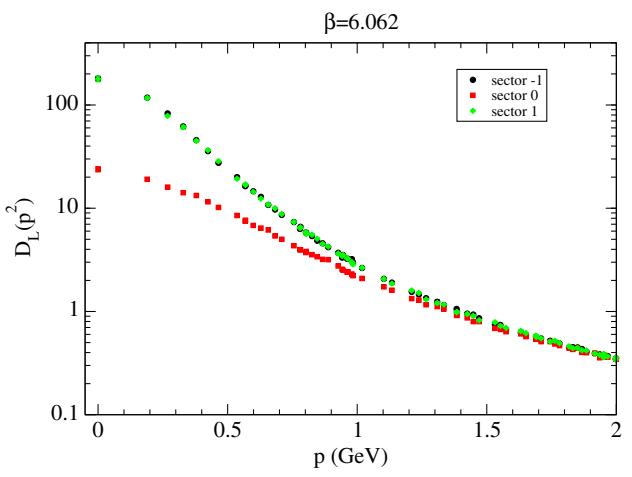

(d) $T=271.9 \mathrm{MeV}$

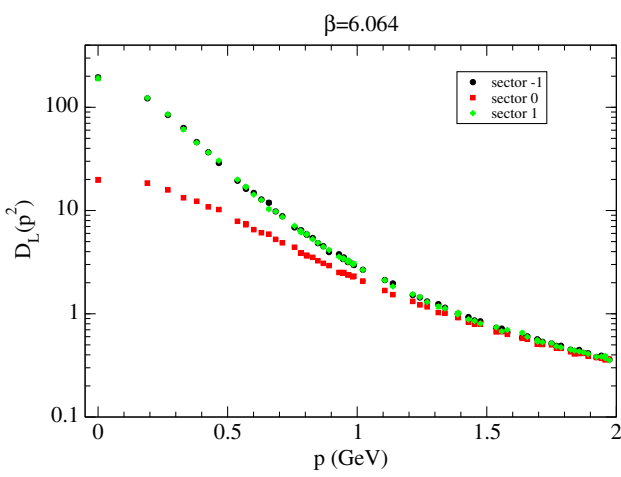

(f) $T=272.9 \mathrm{MeV}$

FIG. 8. Electric gluon form factor $D_{L}\left(p^{2}, T\right)$ for simulations using the finer $72^{3} \times 8$ lattices.

(blue lines) removing the configurations that, according to our criterion, will not be used to compute the propagators. If one considers all the configurations generated by the sampling, the histograms are spread over much larger values of $|L|$ and often show several maxima. On the other hand, our selection of configurations gives rise to a histogram where the distribution of the values of the Polyakov loop clearly have a single maximum. To illustrate the effects on the gluon propagator, in Fig. 6 we report an example where the longitudinal gluon form factor is shown, before and after performing our selection of configurations. As can be seen, the effect of our selection can be well beyond one standard deviation.

\section{THE GLUON PROPAGATOR NEAR THE PHASE TRANSITION}

As discussed in the last section, the proper computation of the gluon propagator for temperatures near $T_{c}$ needs a selection procedure to separate those configurations which can be associated to the deconfined or confined phase. This has been done by looking at the Monte Carlo time evolution for the Polyakov loop and/or $D_{L}(0)$. Recall that we considered 100 configurations for each simulation, and our selection procedure implies that in several cases we will not use the full set of configurations. In Table II the information of Table I is repeated, but including the number of configurations that survive the selection procedure. 


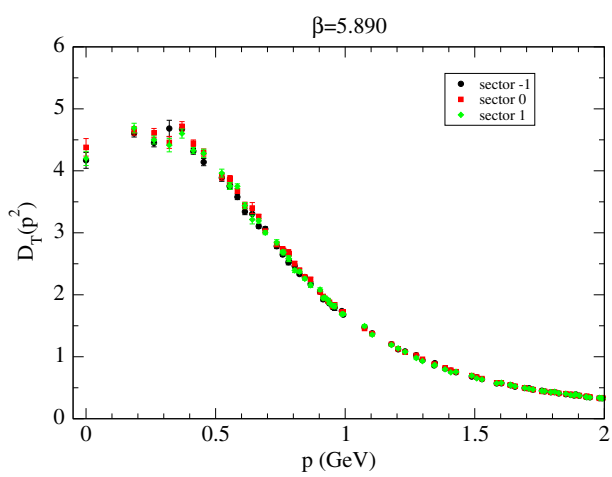

(a) $T=265.9 \mathrm{MeV}$

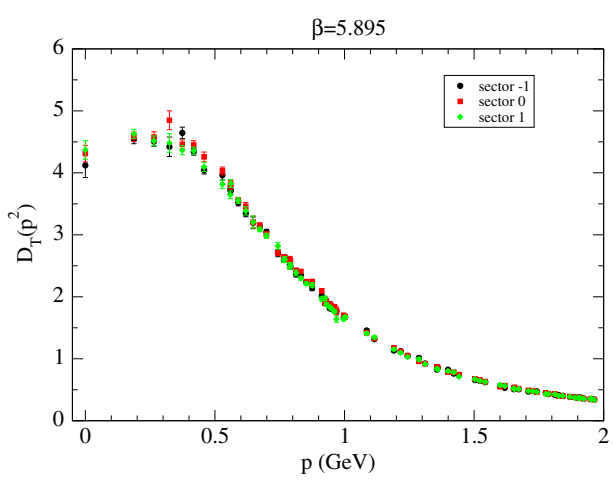

(c) $T=268.5 \mathrm{MeV}$

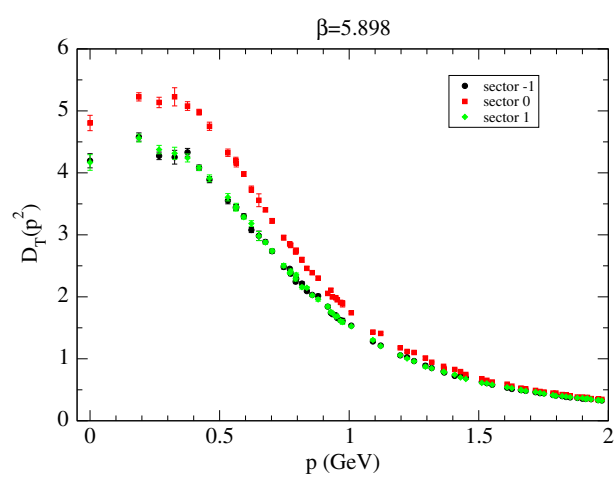

(e) $T=270.0 \mathrm{MeV}$

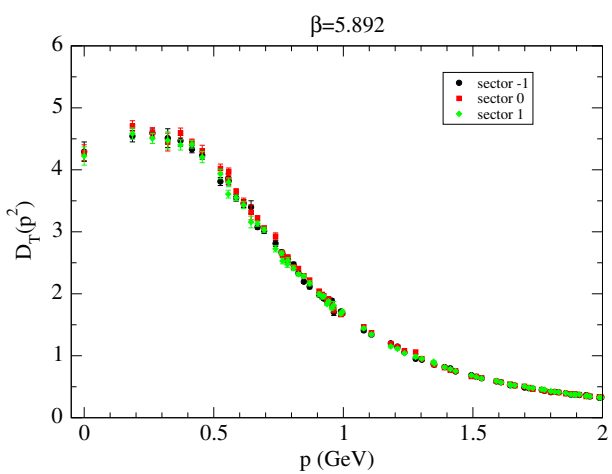

(b) $T=266.9 \mathrm{MeV}$

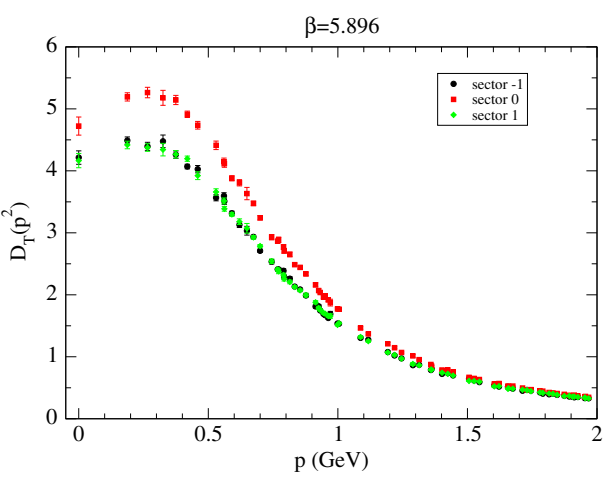

(d) $T=269.0 \mathrm{MeV}$

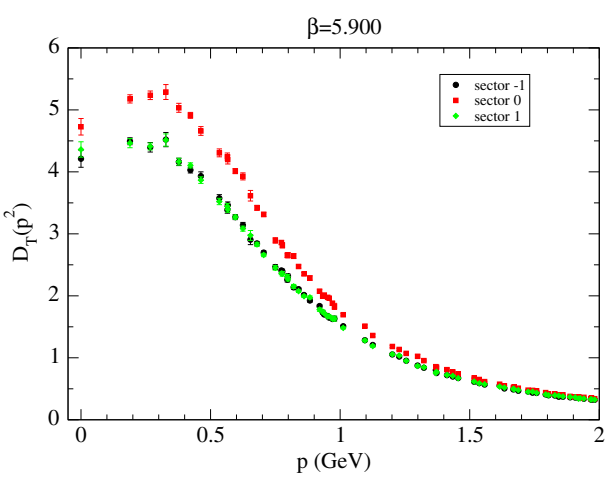

(f) $T=271.0 \mathrm{MeV}$

FIG. 9. Magnetic gluon form factor $D_{T}\left(p^{2}, T\right)$ for simulations using the coarser $54^{3} \times 6$ lattices.

In Figs. 7, 8, 9, and 10 we report on the two gluon form factors for the temperatures reported in Table I and after applying the selection procedure to the gauge configurations. As the figures show, it is in the electric sector where the deconfinement transition has a larger impact. Indeed, for the zero sector $D_{L}\left(p^{2} ; T\right)$ is strongly suppressed in the deconfined phase, while the magnetic sector has a much more modest increase as $\mathrm{T}$ crosses $T_{c}$. This reproduces the behavior already observed in various simulations; see, e.g., Refs. $[8,15]$ and references therein.

Moreover, for $T>T_{c}$ the gluon form factors associated to the various $Z_{3}$ sectors are not only different quantitatively but also qualitatively. This difference is observed only between the 0 sector and the \pm 1 sectors. The longitudinal propagator $D_{L}$ associated with the \pm 1 sectors is strongly enhanced if compared with the zero-sector result. In fact, for the zero sector, $D_{L}$ defines a larger mass scale, compared to the corresponding longitudinal propagators associated with the others sectors. On the other hand, the transverse propagator $D_{T}$ for the 0 sector seems to take higher values, and therefore one can associate a smaller mass scale, in comparison with transverse propagators defined in the \pm 1 sectors.

The separation of $D_{L}$ and $D_{T}$ associated with the various $Z_{3}$ sectors starts around the deconfinement phase transition. 


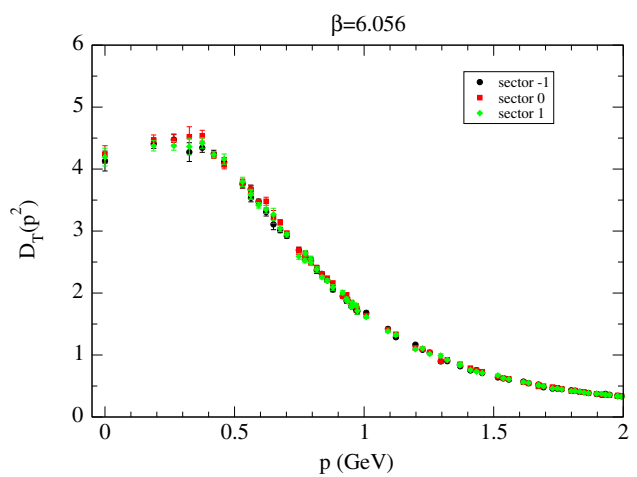

(a) $T=269.2 \mathrm{MeV}$

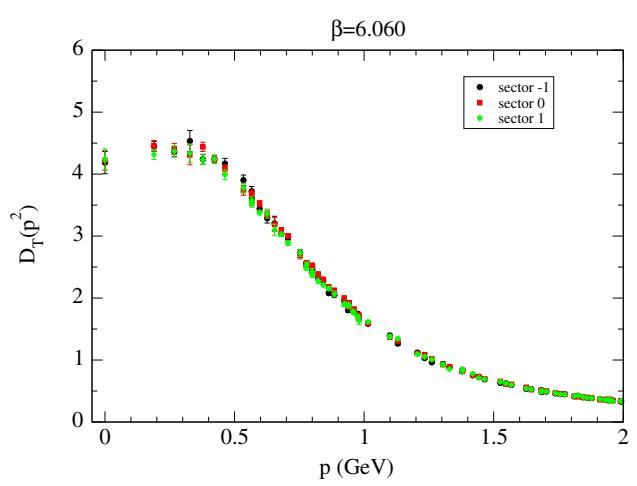

(c) $T=271.0 \mathrm{MeV}$

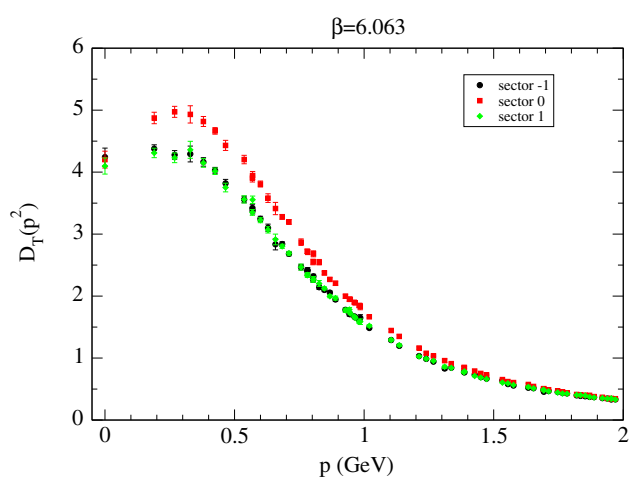

(e) $T=272.4 \mathrm{MeV}$

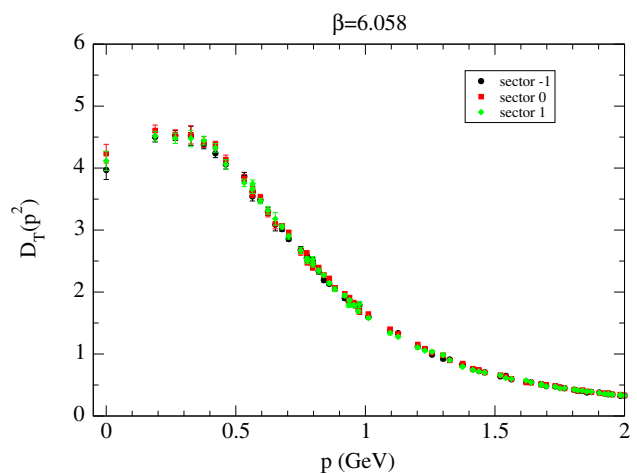

(b) $T=270.1 \mathrm{MeV}$

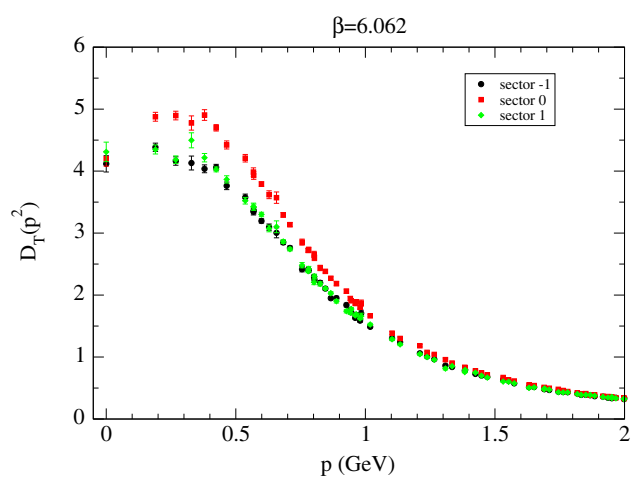

(d) $T=271.9 \mathrm{MeV}$

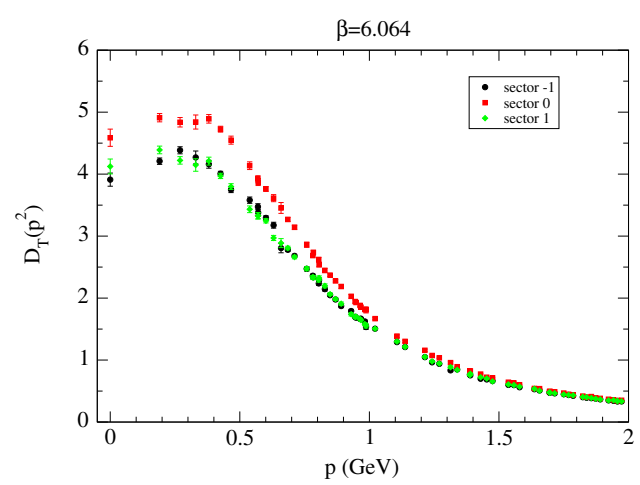

(f) $T=272.9 \mathrm{MeV}$

FIG. 10. Magnetic gluon form factor $D_{T}\left(p^{2}, T\right)$ for simulations using the finer $72^{3} \times 8$ lattices.

For the coarser lattice, this separation starts to show up at $T=267 \mathrm{MeV}$ for $D_{L}$ and it is clearly seen for $T=$ $269 \mathrm{MeV}$ and above. For the magnetic form factor $D_{T}$, the difference between the sectors sets in at $T=269 \mathrm{MeV}$. For the finer lattice, the differences in $D_{L}$ for the various $Z_{3}$ sectors start at $T=270 \mathrm{MeV}$, while $D_{T}$ distinguishes the various sectors for $T=272 \mathrm{MeV}$ and above. From the separation of the gluon form factors one can identify a deconfinement phase transition at $T_{c}=267-272 \mathrm{MeV}$, in agreement with the values quoted in the literature for the pure SU(3) gauge theory.
The observed difference between the propagators for the various $Z_{3}$ sectors can be better illustrated by looking at how $D_{L}(0)$ evolves with the temperature. Furthermore, one can understand the effects of our selection procedure to distinguish the configurations between the different phases by studying $D_{L}(0)$ as a function of the temperature.

In Fig. 11 the zero-momentum electric form factor is plotted for the various temperatures for the coarser and finer lattices. The figure also shows the differences of taking into account all the configurations ("no cuts") and of using our selection procedure. As can be observed, the discontinuity 


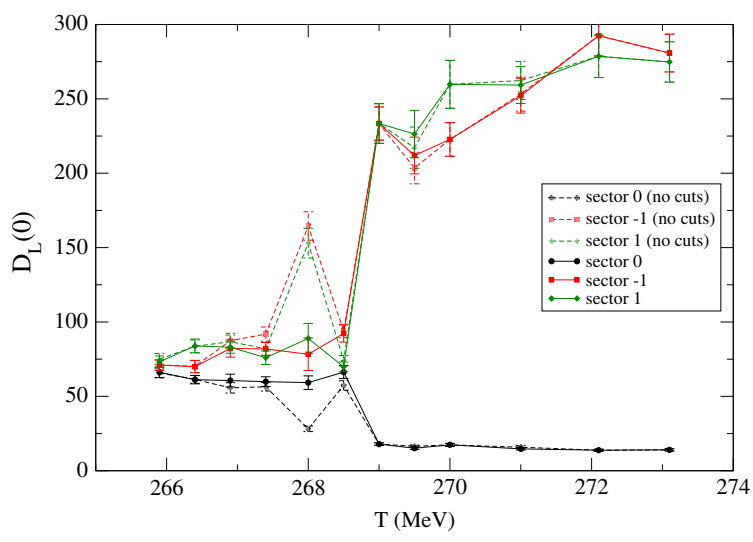

(a) $54^{3} \times 6$ lattices.

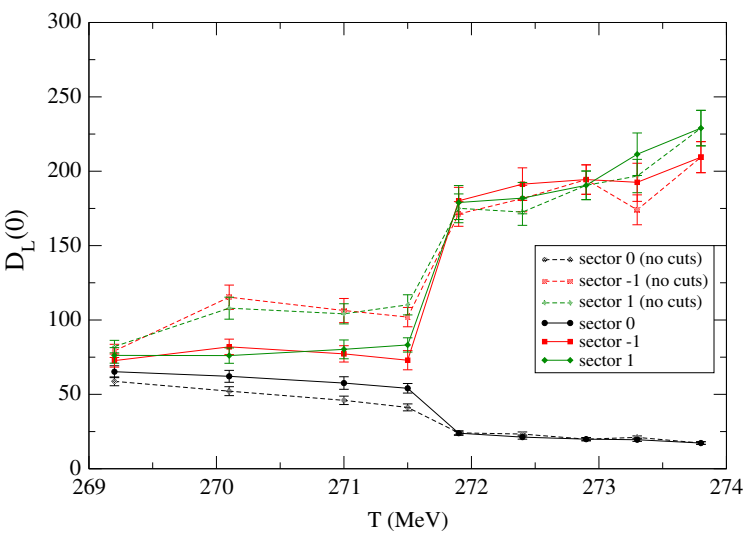

(b) $72^{3} \times 8$ lattices.

FIG. 11. $D_{L}(0)$ as a function of temperature.

in $D_{L}(0)$ at the critical temperature is enhanced when the separation of phases is performed. On the other hand, comparing the coarser and finer lattice results, it seems that the separation of $D_{L}(0)$ between the various sectors above $T_{c}$ is reduced when approaching the continuum limit.

In order to try to understand if the separation vanishes in the continuum limit, we have performed an additional simulation using a $90^{3} \times 10$ lattice and $\beta=6.212$, which has $a=0.07135 \mathrm{fm}$ and $T=276.6 \mathrm{MeV}$. We have checked that this simulation is in the deconfined phase. The computed gluon form factors reproduce the pattern observed and already reported in Figs. 7-9 and 10. In Table III we compare the results for $D_{L}(0)$ and $D_{T}(0)$ for the simulations we have performed just above $T_{c}$. The results suggest that the separation of the electrical gluon form factors observed between the various $Z_{3}$ sectors above $T_{c}$ is not a lattice artifact. From our simulation closer to the continuum one can claim that $\left|D_{L}(0 ; \theta=0)-D_{L}(0 ; \theta= \pm 2 \pi / 3)\right| \approx 180 \mathrm{MeV}^{-2}$, where $\theta$ stands for the phase of the Polyakov loop.

TABLE III. Comparison of $D_{L}(0)$ and $D_{T}(0)$ (in $\mathrm{MeV}^{-2}$ ) for various ensembles just above $T_{c} . \Delta D_{L}(0)$ (errors added in quadrature) refers to the modulus of the difference of $D_{L}(0)$ between the sectors 0 and -1 .

\begin{tabular}{lcccc}
\hline \hline & & $54^{3} \times 6$ & $72^{3} \times 8$ & $90^{3} \times 10$ \\
& Sector & $\beta=5.896$ & $\beta=6.062$ & $\beta=6.212$ \\
$\mathrm{~T}=$ & $269 \mathrm{MeV}$ & $271.9 \mathrm{MeV}$ & $276.6 \mathrm{MeV}$ \\
\hline & & $4.21 \pm 0.11$ & $4.12 \pm 0.13$ & $3.81 \pm 0.13$ \\
$D_{T}(0)$ & 0 & $4.72 \pm 0.15$ & $4.20 \pm 0.12$ & $4.51 \pm 0.13$ \\
& 1 & $4.17 \pm 0.13$ & $4.31 \pm 0.16$ & $4.04 \pm 0.10$ \\
& -1 & $233 \pm 11$ & $180.1 \pm 9.0$ & $203 \pm 12$ \\
$D_{L}(0)$ & 0 & $17.92 \pm 0.84$ & $23.9 \pm 1.4$ & $19.3 \pm 1.0$ \\
& 1 & $233 \pm 13$ & $179 \pm 11$ & $172.9 \pm 9.3$ \\
$\Delta D_{L}(0)$ & & $215 \pm 11$ & $156.2 \pm 9.1$ & $184 \pm 12$ \\
\hline \hline
\end{tabular}

\section{SUMMARY AND CONCLUSIONS}

In this paper, we have investigated the correlations between the modulus of the Polyakov loop, its phase, and the Landau gauge gluon propagator at finite temperature for pure Yang-Mills SU(3) theory. In accordance with the literature, for temperatures above the deconfinement transition, the simulations show that the center symmetry is spontaneously broken and the time history of the Monte Carlo reveals that the phase of the Polyakov loop is always close to $\theta \approx 0, \pm 2 \pi / 3$. For temperatures below $T_{c}$, the Monte Carlo time history shows that $|L| \approx 0$.

We also discussed the computation of the gluon field and the gluon propagator for lattice configurations such that the phase of the Polyakov loop is $\theta \neq 0$. Our analysis shows that the usual definition given in Eq. (5) provides a valid way of computing the gluon field from the links and, therefore, the gluon propagator.

For temperatures above $T_{c}$, our simulations show that the gluon propagator associated to configurations with $\theta \approx 0$ and $\theta \approx \pm 2 \pi / 3$ differs quantitatively and qualitatively. For $T<T_{c}$, this difference is not observed. Therefore, the difference on the propagators can be used to identify the phase (confined or deconfined) of a given configuration. Indeed, this difference behaves as an order parameter for the confinement-deconfinement transition, vanishing for $T<T_{c}$ and taking finite nonzero values for $T>T_{c}$.

Although in the current work we have been concerned with pure Yang-Mills theory, the situation described in the previous paragraph for the gluon propagator-namely, the inequivalence between the zero sector, where $\theta \approx 0$, and \pm 1 sectors, where $\theta \approx \pm 2 \pi / 3$ — can also be observed in full QCD. Indeed, in Ref. [57] the authors relied on full QCD supplemented by a gluon mass term to investigate the effective potential as a function of the temperature $T$, of the chemical potential $\mu$, and of a purely imaginary chemical potential $i \mu$. For the latter case of a purely imaginary chemical potential, it was observed that the effective 


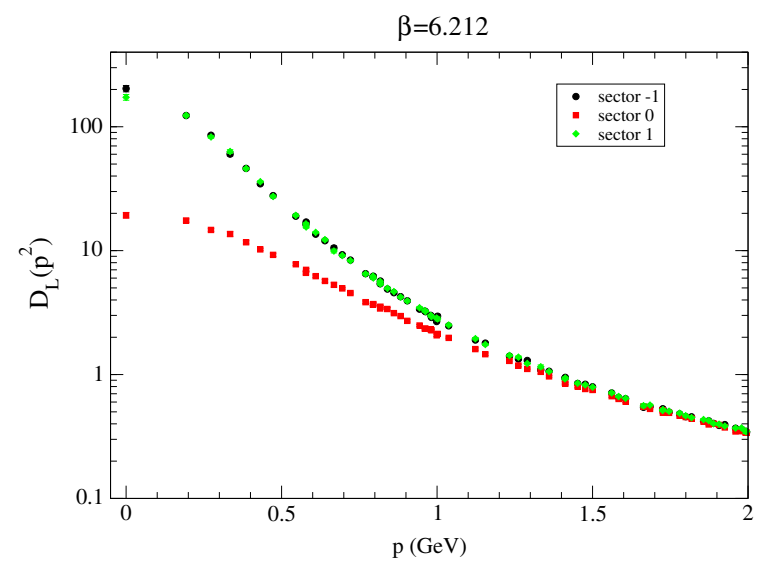

(a) Longitudinal component.

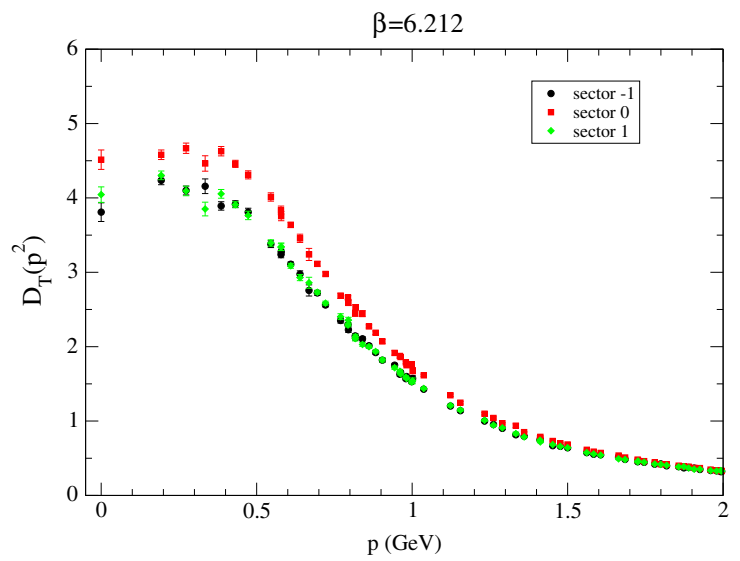

(b) Transverse component.

FIG. 12. Results for a simulation on a $90^{3} \times 10$ lattice, for $\beta=6.212$.

potential distinguishes the various possible $\theta$ and the $Z_{3}$ sectors are not related by symmetry transformations.

In what concerns the gluon propagator form factors above $T_{c}$, we observed a huge enhancement of the electric form factor and a suppression of the magnetic form factor for configurations where $\theta \approx \pm 2 \pi / 3$ relative to those where $\theta \approx 0$. Once more, the simulations show that it is in the electric sector where the dynamics is more sensitive to the deconfinement transition.

The pure Yang-Mills SU(3) theory has a first-order transition to the deconfinement phase and the simulations performed for temperatures near the critical temperature required a careful analysis. Relying on the difference of the propagators associated to the various values of the phase of the Polyakov loop, we showed that it is possible to identify the phase (confined or deconfined) of a given configuration. Indeed, the criterion seems to be able to separate the configurations in each phase (see Fig. 4), and this separation impacts directly on the computation of the propagator for temperatures near $T_{c}$ (see Fig. 11). In fact, the effects of taking into account the configurations in either phase can be misunderstood as finite-volume/-size effects. For example, the "systematic effects" reported in a recent analysis of the SU(2) gluon propagator close to the critical temperature [47] are possibly due to the mixing between the different nature of the gauge configurations generated by the Monte Carlo. Note also that, as discussed in Sec. IV (see Table III and Fig. 12), the observed difference between the gluon form factors computed in different sectors seems to survive in the continuum limit.

The criterion to separate confined and deconfined phases based on the differences of the gluon propagator associated with the various $\theta$ values also allowed us to estimate the critical temperature $T_{c}$ in the range 269-272 MeV, in good agreement with the literature.

The gluon propagator is not a renormalization-groupinvariant quantity and the difference between the various sectors observed in the infrared region depends on the renormalization scale. In the current work, all the data was renormalized at $\mu=4 \mathrm{GeV}$ and the differences are clearly seen in the infrared region. These differences in the propagators associated with the various $\theta$ are both quantitative and qualitative. In principle, one could choose a different renormalization scale, e.g., in the infrared region, and, in this case, the differences in the propagators would appear in the ultraviolet region and all the considerations discussed would still apply but for this region of momenta.

In the near future, we plan to extend our simulations to cover a wide range of temperatures to provide a clear picture on the behavior of the various $D_{L}, D_{T}$ and differences between the $Z_{3}$ sectors in a wide range of temperatures. Furthermore, we are aware that the real world contains quark degrees of freedom and the center symmetry is no longer a valid symmetry of the theory. However, for full QCD the behavior of the Polyakov loop as a function of the temperature is similar to the pure Yang-Mills case and, therefore, it would also be interesting to check how the scenario discussed here changes when the quark degrees of freedom are taken into account.

\section{ACKNOWLEDGMENTS}

We acknowledge the Laboratory for Advanced Computing at University of Coimbra for providing HPC computing resources (Milipeia, Centaurus and Navigator) that have contributed to the research results reported within this paper (URL http://www.lca.uc.pt). We acknowledge that the results of this research have been achieved using the PRACE-3IP project (FP7 RI-312763) resource Sisu based in Finland at CSC. The use of Sisu has been provided under DECI-12 project COIMBRALATT2. P. J. Silva acknowledges support by F. C. T. under contract SFRH/BPD/40998/ 2007. This work was supported by Projects No. CERN/FP/ 123612/2011, No. CERN/FP/123620/2011 and No. PTDC/ FIS/100968/2008, projects developed under initiative QREN financed by UE/FEDER through Programme COMPETE. 
[1] C Roland, Int. J. Mod. Phys. A 30, 1546010 (2015).

[2] T. A. Trainor, Int. J. Mod. Phys. E 23, 1430011 (2014).

[3] H. B. Meyer, arXiv:1512.06634.

[4] C. Gattringer, Proc. Sci., LATTICE 2013 (2013) 002.

[5] Y. Iwasaki, K. Kanaya, T. Yoshié, T. Hoshino, T. Shirakawa, Y. Oyanagi, S. Ichii, and T. Kawai, Phys. Rev. D 46, 4657 (1992).

[6] G. Boyd, J. Engels, F. Karsch, E. Laermann, C. Legeland, M. Lütgemeier, and B. Petersson, Nucl. Phys. B469, 419 (1996).

[7] B. Lucini, M. Teper, and U. Wenger, J. High Energy Phys. 01 (2004) 061.

[8] P. J. Silva, O. Oliveira, P. Bicudo, and N. Cardoso, Phys. Rev. D 89, 074503 (2014).

[9] T. Bhattacharya et al. (HotQCD Collaboration), Phys. Rev. Lett. 113, 082001 (2014).

[10] Y. Aoki, G. Endrodi, Z. Fodor, S. Katz, and K. Szabo, Nature (London) 443, 675 (2006).

[11] S. Borsanyi, Z. Fodor, C. Hoelbling, S. D. Katz, S. Krieg, C. Ratti, and K. K. Szabo (Wuppertal-Budapest Collaboration), J. High Energy Phys. 09 (2010) 073.

[12] A. Bazavov et al., Phys. Rev. D 85, 054503 (2012).

[13] J. I. Kapusta and C. Gale, Finite-Temperature Field Theory: Principles and Applications (Cambridge University Press, Cambridge, England, 2011).

[14] P. M. Lo, B. Friman, O. Kaczmarek, K. Redlich, and C. Sasaki, Phys. Rev. D 88, 074502 (2013).

[15] R. Aouane, V. G. Bornyakov, E.-M. Ilgenfritz, V. K. Mitrjushkin, M. Müller-Preussker, and A. Sternbeck, Phys. Rev. D 85, 034501 (2012).

[16] V. N. Gribov, Nucl. Phys. B139, 1 (1978).

[17] N. Vandersickel and D. Zwanziger, Phys. Rep. 520, 175 (2012).

[18] A. Cucchieri, Nucl. Phys. B508, 353 (1997).

[19] P. J. Silva and O. Oliveira, Proc. Sci., Lattice 2010 (2010) 287, arXiv:1011.0483.

[20] P. J. Silva and O. Oliveira, Proc. Sci., LATTICE 2007 (2007) 333, arXiv:0710.0669.

[21] P. J. Silva and O. Oliveira, Nucl. Phys. B690, 177 (2004).

[22] A. Sternbeck and M. Muller-Preussker, Phys. Lett. B 726, 396 (2013).

[23] C. Hong-Mo and T. Sheung Tsun, Some Elementary Gauge Theory Concepts (World Scientific, Singapore, 1993).

[24] C. Gattringer, Phys. Lett. B 690, 179 (2010).

[25] C. Gattringer and A. Schmidt, J. High Energy Phys. 01 (2011) 051.

[26] F. M. Stokes, W. Kamleh, and D. B. Leinweber, Ann. Phys. (Amsterdam) 348, 341 (2014).

[27] G. Endrödi, C. Gattringer, and H.-P. Schadler, Phys. Rev. D 89, 054509 (2014).

[28] M. Asakawa, S. A. Bass, and B. Müller, Phys. Rev. Lett. 110, 202301 (2013).

[29] V. M. Belyaev, I. I. Kogan, G. W. Semenoff, and N. Weiss, Phys. Lett. B 277, 331 (1992).
[30] A. V. Smilga, Ann. Phys. (N.Y.) 234, 1 (1994).

[31] S. Chandrasekharan and N. Christ, Nucl. Phys. B, Proc. Suppl. 47, 527 (1996).

[32] S. Chandrasekharan and S. Huang, Phys. Rev. D 53, 5100 (1996).

[33] T. G. Kovács, Proc. Sci., LATTICE 2008 (2008) 198, arXiv:0810.4763.

[34] O. Oliveira and P. J. Silva, Proc. Sci., LATTICE2014 (2014) 355, arXiv:1411.0133.

[35] A. Cucchieri, A. Maas, and T. Mendes, Phys. Rev. D 75, 076003 (2007).

[36] C. S. Fischer, A. Maas, and J. A. Muller, Eur. Phys. J. C 68, 165 (2010).

[37] A. Cucchieri and T. Mendes, Proc. Sci., Lattice 2010 (2010) 280, arXiv:1101.4537.

[38] A. Cucchieri and T. Mendes, Proc. Sci., FacesQCD (2010) 007, arXiv:1105.0176.

[39] V. Bornyakov and V. Mitrjushkin, Phys. Rev. D 84, 094503 (2011).

[40] V. Bornyakov and V. Mitrjushkin, Int. J. Mod. Phys. A 27, 1250050 (2012).

[41] A. Maas, J. M. Pawlowski, L. von Smekal, and D. Spielmann, Phys. Rev. D 85, 034037 (2012).

[42] A. Cucchieri and T. Mendes, Proc. Sci., Lattice 2011 (2011) 206, arXiv:1201.6086.

[43] O. Oliveira and P. J. Silva, Acta Phys. Pol. B Proc. Suppl. 5, 1039 (2012).

[44] O. Oliveira and P. Silva, Proc. Sci., Lattice 2012 (2012) 216, arXiv: 1210.8033 .

[45] P. J. Silva and O. Oliveira, Proc. Sci., Confinement X (2012) 045, arXiv:1301.4094.

[46] A. Maas, Phys. Rep. 524, 203 (2013).

[47] T. Mendes and A. Cucchieri, Proc. Sci., LATTICE 2013 (2013) 456, arXiv:1401.6908.

[48] U. Reinosa, J. Serreau, M. Tissier, and N. Wschebor, Phys. Rev. D 89, 105016 (2014).

[49] U. Reinosa, J. Serreau, M. Tissier, and N. Wschebor, Phys. Lett. B 742, 61 (2015).

[50] U. Reinosa, J. Serreau, M. Tissier, and N. Wschebor, Phys. Rev. D 91, 045035 (2015).

[51] U. Reinosa, J. Serreau, M. Tissier, and N. Wschebor, Phys. Rev. D 93, 105002 (2016).

[52] F. E. Canfora, D. Dudal, I. F. Justo, P. Pais, L. Rosa, and D. Vercauteren, Eur. Phys. J. C 75, 326 (2015).

[53] O. Oliveira and P. J. Silva, Phys. Rev. D 86, 114513 (2012).

[54] R. G. Edwards and B. Joo (SciDAC, LHPC, and UKQCD Collaborations), Nucl. Phys. B, Proc. Suppl. 140, 832 (2005).

[55] C. T. H. Davies, G. G. Batrouni, G. R. Katz, A. S. Kronfeld, G. P. Lepage, K. G. Wilson, P. Rossi, and B. Svetitsky, Phys. Rev. D 37, 1581 (1988).

[56] M. Pippig, SIAM J. Sci. Comput. 35, C213 (2013).

[57] U. Reinosa, J. Serreau, and M. Tissier, Phys. Rev. D 92, 025021 (2015). 